\title{
Atresia Congênita do Tronco da Coronária Esquerda em Adulto Jovem: Rara e Potencialmente Fatal Anomalia Coronária
}

\author{
Leandro Zacarias Figueiredo de Freitas ${ }^{1}$, Marinella Centemero ${ }^{1}$, Ricardo Costa ${ }^{1}$, J. Ribamar Costa Jr. ${ }^{1}$, \\ Fausto Feres', Paulo Paredes Paulista', Marcelo Nakashima', Javier Obregon', Juliana F. Kelendjian', \\ Andréia Maranhão' ${ }^{1}$ Ibraim Masciarelli Pinto ${ }^{1}$, Alexandre Abizaid ${ }^{1}$,
}

Amanda G. M. R. Sousa ${ }^{1}$, J. Eduardo Sousa ${ }^{1}$

\section{RESUMO}

Relato do caso de paciente do sexo masculino, 35 anos de idade, com atresia congênita do tronco da artéria coronária esquerda, afecção extremamente rara e com poucos casos descritos na literatura. Nessa afecção, o óstio da coronária esquerda está ausente, o tronco da coronária esquerda termina em fundo cego e o fluxo sanguíneo se dá da coronária direita para a esquerda por pequenas artérias colaterais e retrogradamente. O diagnóstico diferencial envolve outras malformações congênitas (artéria coronária direita única e origem anômala da artéria coronária esquerda do tronco da artéria pulmonar) e doença aterosclerótica adquirida do tronco da coronária esquerda.

DESCRITORES: Cardiopatias congênitas. Vasos coronários. Doenças das coronárias. Revascularização miocárdica.

\section{A} atresia do tronco da coronária esquerda é uma rara anomalia coronária na qual não se identifica o óstio da coronária esquerda e a porção proximal do tronco termina abruptamente. Dessa forma, a coronária esquerda apresenta fluxo retrógrado a partir de colaterais provenientes da coronária direita. A circulação colateral que nutre a coronária esquerda parte em geral da artéria do cone e anastomoses intrasseptais, ventriculares anteriores e posteriores.

1 Instituto Dante Pazzanese de Cardiologia - São Paulo, SP, Brasil. Correspondência: Leandro Zacarias Figueiredo de Freitas. Rua 16-A, 627/402 - Aeroporto - Goiânia, GO, Brasil - CEP 74075-150 E-mail: leandrozffreitas@hotmail.com

Recebido em: 12/1/2011 • Aceito em: 9/3/2011

\section{ABSTRACT}

Congenital Atresia of the Left Main Coronary Artery in a Young Adult: A Rare and Potentially Fatal Coronary Anomaly

We report the case of a 35-year-old male patient with congenital atresia of the left main coronary artery, a rare disease with few cases reported in the literature. In this anomaly, the left coronary ostium is absent, the proximal left main trunk ends blindly and blood flow is retrograde from the right to the left coronary artery by small collateral arteries. Differential diagnosis includes other congenital malformations (single right coronary artery and anomalous origin of the left coronary artery from the pulmonary artery) and acquired atherosclerotic disease of the left main coronary artery.

KEY-WORDS: Heart defects, congenital. Coronary vessels. Coronary disease. Myocardial revascularization.

A atresia pode ser verdadeira ou o tronco pode ser hipoplásico. De qualquer forma, a afecção é grave e pode levar o paciente à morte. Essa afecção é classificada nas formas infantil e adulta. Na forma infantil, coexistem outros defeitos cardíacos, entre os quais o mais frequente é a estenose aórtica supravalvar. Na forma adulta, em geral não se detectam anomalias associadas.

Relatamos a seguir um caso de apresentação tardia da doença (forma adulta).

\section{RELATO DO CASO}

Paciente de 35 anos, sexo masculino, branco, casado, natural e procedente de São Paulo (SP, Brasil), previamente assintomático. Esportista até os 34 anos de idade, quando, por motivos profissionais, interrompeu a prática de atividade física. Um ano após, por desejar retomar a prática esportiva e considerando-se 
com pouca disposição para a realização de grandes esforços, resolveu procurar o médico para exames de rotina. Na ocasião o paciente não apresentava histórico familiar para doenças cardiovasculares.

Foram solicitados exames laboratoriais e teste ergométrico. Os primeiros revelaram hipertrigliceridemia (301 mg/dl) e hipercolesterolemia (240 mg/dl), com fração de lipoproteína de baixa densidade (LDL) de $153 \mathrm{mg} / \mathrm{dl}$ e de lipoproteína de alta densidade (HDL) de $26 \mathrm{mg} / \mathrm{dl}$. A glicemia era normal (86 mg/dl).

No teste ergométrico (protocolo Ellestad), o paciente apresentou, no esforço submáximo, infradesnivelamento do segmento ST de $2 \mathrm{~mm}$ na derivação MC5 (caráter ascendente lento) e infradesnivelamento de menor magnitude nas derivações D2, D3, AVF, V4, V5 e V6 no pico do esforço. Durante a recuperação, identificou-se a retificação do segmento ST, com recuperação lenta deste ( $>6$ minutos) no repouso. O paciente alcançou volume máximo de oxigênio $\left(\mathrm{VO}_{2} \max \right)$ de $40,42 \mathrm{ml} / \mathrm{kg} / \mathrm{min}$ e $11,6 \mathrm{MET}$.

Outros exames complementares, como ecocardiografia transtorácica em repouso e cintilografia de perfusão miocárdica (estresse e repouso), foram solicitados.

A ecocardiografia revelou cavidades atriais e ventriculares com diâmetros normais, assim como funções sistólica (fração de ejeção $=70 \%$ ) e diastólica de ventrículo esquerdo preservadas, além de ausência de sinais de disfunção contrátil segmentar. Na cintilografia de perfusão miocárdica associada ao teste ergométrico (protocolo Bruce, no qual o paciente atingiu o esforço submáximo), verificou-se presença de hipoconcentração transitória de leve intensidade nas paredes anterior (porções média e apical) e apical de ventrículo esquerdo, função ventricular esquerda global preservada, e comprometimento segmentar das paredes anterior (porções média e apical) e apical após estresse.

Em decorrência desses achados, o paciente foi encaminhado para realização de cinecoronariografia, cujos resultados demonstraram (Figura 1):

1) coronária direita sem evidências de processo ateromatoso significativo (durante a realização do exame verificou-se presença de espasmo coronário em sua porção proximal, que cedeu após a administração de nitratos);

2) extensa rede colateral da coronária direita para a coronária esquerda;

3) dilatação do ramo do cone, com origem no seio coronário direito em óstio da coronária direita, emitindo colateral para o terço médio da artéria descendente anterior, identificando-se enchimento retrógrado dessa artéria (a artéria descendente anterior apresentava calibre reduzido nas porções média e distal, observando-se também opacificação retrógrada da artéria circunflexa, que se apresentava isenta de processo ateromatoso significativo), sem visualização do tronco da artéria pulmonar; e

4) aorta ascendente de calibre habitual, valva aórtica tricúspide competente, não se observando opacificação anterógrada da coronária esquerda a partir do seio de Valsalva esquerdo.

Após a realização da cinecoronariografia, com o intuito de avaliar melhor a anatomia coronária e de complementar os dados obtidos, o paciente foi submetido a angiotomografia das artérias coronárias. O exame revelou opacificação da coronária esquerda, porém não se verificou conexão entre a aorta e as artérias descendente anterior e circunflexa. Paralelamente não foram detectados sinais de trombose ou irregularidades na raiz da aorta que pudessem sugerir oclusão do tronco da coronária esquerda.

Outro achado bastante interessante da angiotomografia, também revelado pela cinecoronariografia, diz respeito à presença da extensa rede de colaterais provenientes da coronária direita, por meio do anel de Vieussens, permitindo o fluxo de sangue para os leitos vasculares das artérias descendente anterior e circunflexa (Figura 2). Também foi possível detectar que as porções média e distal da descendente anterior eram de fino calibre, com redução luminal significativa e ausência de ateromatose relevante ao exame. Além disso, o menor realce das porções subendocárdicas das paredes anterior e apical detectadas pela angiotomografia sugere fortemente a presença de atresia do tronco da coronária esquerda.

Após discussão do caso, foi indicada a realização de cirurgia de revascularização miocárdica. A aorta foi aberta transversalmente, não sendo visualizado o óstio da coronária esquerda. A equipe cirúrgica optou pela revascularização miocárdica com uso de artéria torácica interna esquerda para ramo diagonal, em decorrência da perda acentuada do calibre da artéria descendente anterior a partir de seu terço médio, confirmando os achados identificados tanto pela cinecoronariografia como pela angiotomografia (Figura 3).

O paciente não apresentou quaisquer intercorrências durante a cirurgia e no pós-operatório, recebendo alta no sétimo dia em uso de ácido acetilsalicílico e estatina. O paciente permanecia assintomático após um ano de seguimento.

\section{DISCUSSÃO}

A atresia congênita do tronco da coronária esquerda é uma doença extremamente rara, com poucos relatos na literatura. Admite-se incidência de aproximadamente $0,01 \%$ a $0,04 \%$ em estudos de necropsia. ${ }^{1} \mathrm{~A}$ literatura é bastante escassa sobre esse tipo de anomalia, trazendo dificuldade na padronização terapêutica e no conhecimento do prognóstico a longo prazo. 


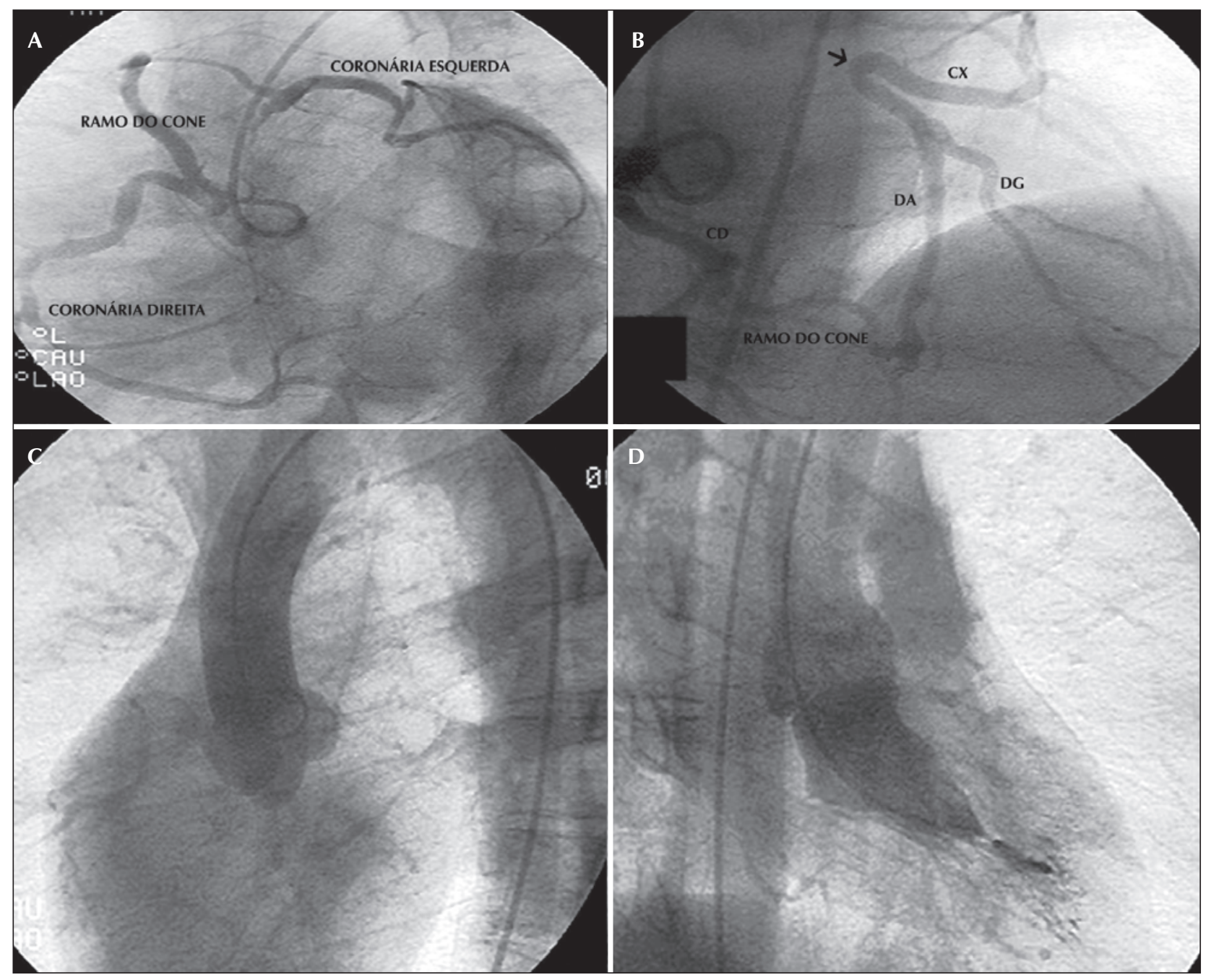

Figura 1 - Em A, opacificação seletiva da artéria coronária direita revelando presença de ectasia de ramo do cone suprindo a circulação coronária esquerda. Em B, circulação colateral da coronária direita para a descendente anterior (a seta indica o término abrupto do tronco da coronária esquerda). Em C, aortografia demonstrando opacificação normal não-seletiva da coronária direita e ausência de opacificação da coronária esquerda, apesar da presença do seio de Valsalva esquerdo. Em D, ventriculografia demonstrando função ventricular esquerda normal. $C X=$ artéria circunflexa; $D A=$ artéria descendente anterior; $D G=$ ramo diagonal.

Seu quadro clínico costuma ser subdividido nas formas infantil e adulta. A presença de circulação colateral entre as coronárias direita e esquerda é fundamental na determinação do momento da apresentação clínica. Na forma adulta, como ilustrado no caso relatado, o paciente pode permanecer oligoassintomático com presença de isquemia detectada por meio de provas funcionais, como cintilografia associada a esforço físico. Entretanto, em alguns casos, pode se manifestar na forma de angina ou mesmo morte súbita.

Apesar de, teoricamente, o diagnóstico de atresia do tronco da coronária esquerda poder ser obtido pela ecocardiografia com auxílio do Doppler, o estudo hemodinâmico é fundamental para confirmação diagnóstica. O achado fundamental é a presença de circulação colateral proveniente da coronária direita, permitin- do o enchimento retrógrado da coronária esquerda. A aortografia revela ausência de enchimento anterógrado da coronária esquerda a partir do seio de Valsalva esquerdo.

A tomografia com múltiplos detectores é extremamente útil no diagnóstico, por complementar os achados da cinecoronariografia e demonstrar a ausência de conexão entre a aorta e a coronária esquerda ou eventualmente revelar uma pequena estrutura em forma de cordão no sítio do tronco da coronária esquerda. ${ }^{2}$

A atresia do tronco da coronária esquerda deve ser diferenciada de outras duas malformações congênitas (artéria coronária direita única e origem anômala da artéria coronária esquerda do tronco da artéria pulmonar) e doença aterosclerótica adquirida do tronco da coronária esquerda. A artéria coronária direita 


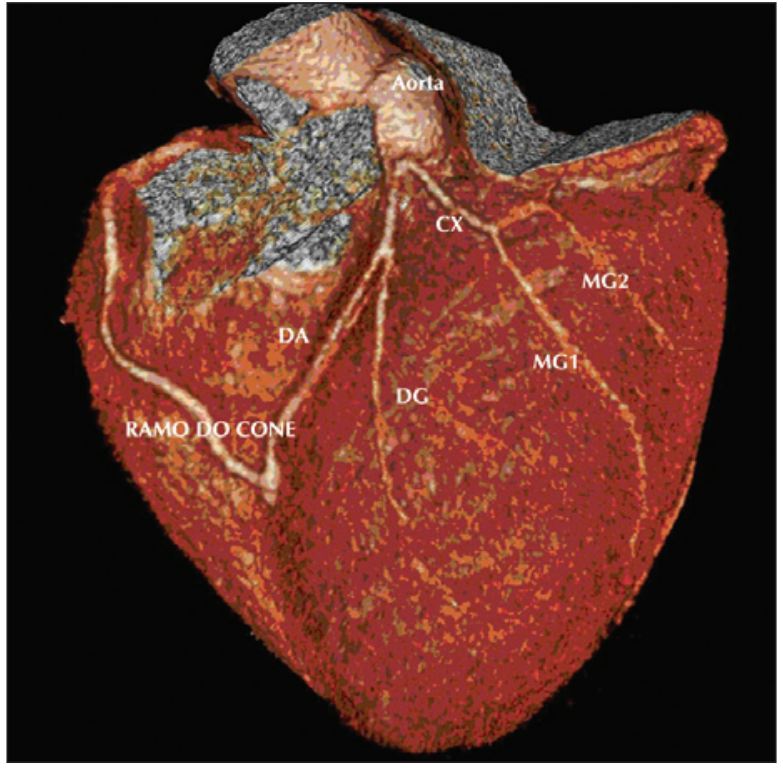

Figura 2 - Angiotomografia de coronárias demonstrando presença de circulação colateral da coronária direita para a descendente anterior e para a circunflexa, sem opacificação do tronco da coronária esquerda. Observa-se, também, redução significativa do calibre das porções média e distal da descendente anterior e nenhuma evidência de doença aterosclerótica. $C X=$ artéria circunflexa; $D A=$ artéria descendente anterior; $\mathrm{DG}=$ ramo diagonal; $\mathrm{MG} 1=$ primeiro ramo marginal; MG2 = segundo ramo marginal.

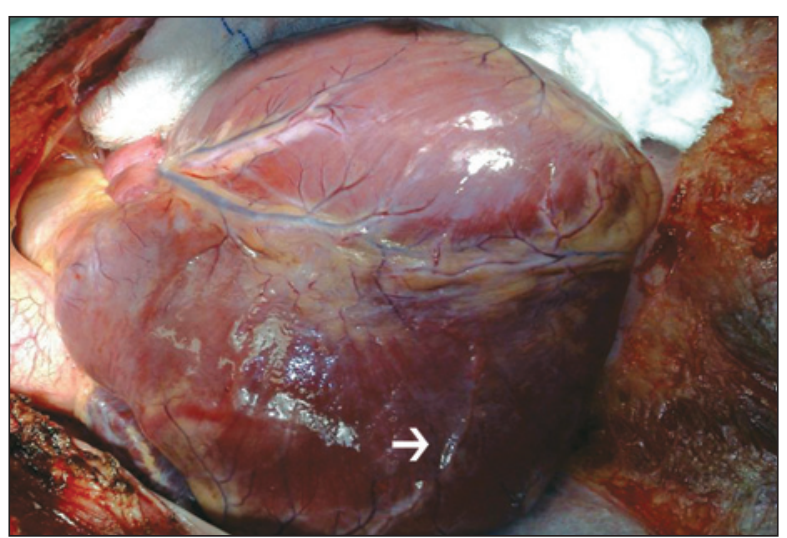

Figura 3 - Coração exposto (vista anterior), evidenciando-se as artérias descendente anterior e primeiro ramo diagonal. A seta indica o ramo do cone dilatado conectando as artérias coronárias direita e esquerda.

única é uma anomalia bem tolerada, na qual toda a circulação coronária é dependente da coronária direita e não está associada a isquemia. Nela o sangue flui da aorta para a periferia por meio de vasos de tamanhos progressivamente menores (padrão centrífugo). Na atresia do óstio do tronco da coronária esquerda, a circulação na coronária esquerda é reversa e o sangue flui da coronária direita para vasos progressivamente maiores da coronária esquerda (padrão centrípeto). A coronariografia permite a fácil diferenciação dessas afecções.

Na origem anômala da artéria coronária esquerda do tronco da artéria pulmonar, o fluxo na coronária esquerda também é reverso. Nessa afecção, a opacificação retrógrada da artéria pulmonar, através da coronária esquerda, pode ser notada durante a angiografia seletiva da artéria coronária direita, como também o fluxo diastólico para a coronária esquerda, durante a angiografia pulmonar.

No adulto, a diferenciação com a oclusão aterosclerótica do tronco da coronária esquerda é difícil, mesmo com a realização de cinecoronariografia e tomografia computadorizada das artérias coronárias. O diagnóstico definitivo só pode ser realizado no intraoperatório ao se observar o tamanho e a aparência externa do tronco da coronária esquerda, que é normal na doença aterosclerótica, mas de fino calibre e fibrótico na atresia do óstio do tronco da coronária esquerda. Nesse caso, o óstio da coronária esquerda também não é detectado.

Em relação ao tratamento dessa afecção, os dados da literatura sugerem que a cirurgia de revascularização miocárdica é a melhor opção, com o objetivo de reduzir a isquemia, preservar a função ventricular e prevenir a ocorrência de morte súbita mesmo em pacientes pouco sintomáticos. ${ }^{3}$ A técnica cirúrgica mais empregada nesses casos consiste na realização do implante da artéria torácica interna esquerda para a artéria descendente anterior ou ramo diagonal, quando aquela não apresentar leito distal adequado para a anastomose..$^{2,4}$ A artéria torácica interna é considerada o enxerto ideal em decorrência das altas taxas de patência a longo prazo, fartamente comprovadas na literatura. ${ }^{2}$ No entanto, por ser tratar de uma doença bastante rara, a casuística é pequena nos diversos serviços, com necessidade de seguimento a longo prazo para se documentar o real benefício dessa cirurgia.

\section{CONFLITO DE INTERESSES}

Os autores declaram não haver conflito de interesses relacionado a este manuscrito.

\section{REFERÊNCIAS}

1. Sharbaugh AH, White RS. Single coronary artery. Analysis of the anatomic variation, clinical importance, and report of five cases. JAMA. 1974;230(2):243-6.

2. Koh E, Nakagawa M, Hamaoka K, Sawada T, Oga K. Congenital atresia of the left coronary ostium: diagnosis and surgical treatment. Pediatr Cardiol. 1989;10(3):159-62.

3. van der Hauwaert LG, Dumoulin M, Moerman P. Congenital atresia of left coronary ostium. Br Heart J. 1982;48(3):298-300.

4. Serraf A, Baron O, Nottin E, Lacour-Gayet F, Bruniaux J, Sousa Uva $M$, et al. Atrésie ou sténose congénitale de l'ostium coronaire gauche. Revascularisation myocardique chez 5 enfants. Arch Mal Coeur Vaiss. 1993;86(5):587-91. 